\title{
A remarkable recurrent nova in $M$ 31: The optical observations
}

\author{
M. J. Darnley ${ }^{1}$, S. C. Williams ${ }^{1}$, M. F. Bode ${ }^{1}$, M. Henze ${ }^{2}$, J.-U. Ness ${ }^{2}$, A. W. Shafter ${ }^{3}$, K. Hornoch ${ }^{4}$, and V. Votruba \\ 1 Astrophysics Research Institute, Liverpool John Moores University, IC2 Liverpool Science Park, Liverpool, L3 5RF, UK \\ e-mail: M. J .Darnley@ljmu.ac.uk \\ 2 European Space Astronomy Centre, PO Box 78, 28691 Villanueva de la Cañada, Madrid, Spain \\ 3 Department of Astronomy, San Diego State University, San Diego CA 92182, USA \\ 4 Astronomical Institute, Academy of Sciences, 25165 Ondřejov, Czech Republic
}

Received 13 January 2014 / Accepted 10 February 2014

\section{ABSTRACT}

\begin{abstract}
Context. In late Nov. 2013 the fifth eruption in five years of the M31 recurrent nova M31N 2008-12a was announced. Aims. In this Letter we address the optical lightcurve and progenitor system of M31N 2008-12a.

Methods. Optical imaging data of the 2013 eruption from the Liverpool Telescope, La Palma; Danish $1.54 \mathrm{~m}$ Telescope, La Silla; and archival Hubble Space Telescope near-IR, optical, and near-UV data are astrometrically and photometrically analysed.

Results. Photometry of the 2013 eruption, combined with three previous eruptions, enabled construction of a template lightcurve of a very fast nova $\left(t_{2}(V) \simeq 4\right.$ days). The archival data allowed recovery of the progenitor system in optical and near-UV data, indicating a red-giant secondary with bright accretion disk, or alternatively a system with a sub-giant secondary but dominated by a disk.

Conclusions. The eruptions of M 31N 2008-12a, and a number of historic X-ray detections, indicate a unique system with a recurrence timescale of $\sim 1$ yr. This implies the presence of a very high-mass white dwarf and a high accretion rate. The recovered progenitor system is consistent with such an elevated rate of accretion. We encourage additional observations, especially towards the end of 2014.
\end{abstract}

Key words. galaxies: individual: M 31 - novae, cataclysmic variables - stars: individual: M31N 2008-12a

\section{Introduction}

Classical and recurrent novae $(\mathrm{CNe}$ and $\mathrm{RNe})$ are cataclysmic variable stars that exhibit eruptions driven by a thermonuclear runaway on the surface of a white dwarf (WD; the primary) in an interacting binary system. Classical nova systems typically contain main sequence secondaries with expected recurrence times of a few $\times 10^{3}-10^{6}$ years (see Bode \& Evans 2008, for recent reviews). Recurrent novae are observed to recur on timescales of ten to a hundred years and most contain evolved, sub-giant or red giant secondaries (SG- and RG-novae, respectively; Darnley et al. 2012).

While hundreds of Galactic CNe are known, there are only ten confirmed RNe in the Milky Way. With over 900 novae discovered in M 31 (Pietsch et al. 2007, and on-line database ${ }^{1}$ ) and with a nova rate of $65_{-15}^{+16} \mathrm{yr}^{-1}$ (Darnley et al. 2006), M31 remains a potentially huge untapped resource for identifying $\mathrm{RNe}$ with only a handful of candidate systems known.

The RN M 31N 2008-12a has been discovered in eruption five times over a five-year period, in 2008, 2009, 2011, 2012, and most recently in Nov 2013. For comparison, the shortest inter-eruption time for a Galactic RN is eight years in the case of U Sco (Schaefer 2010). Eruptions of the system have also been detected in X-rays at entirely separate epochs, an overview of which is given in the accompanying Letter by Henze et al. (2014; hereafter HND2014). Here we briefly describe the optical discoveries up to and including the 2012 eruption; the Dec. 2009 eruption is described in full in Tang et al. (2014).

Nishiyama and Kabashima discovered a M31 nova candidate (M31N 2008-12a) with an unfiltered magnitude of 18.7 at $0^{\mathrm{h}} 45^{\mathrm{m}} 28^{\mathrm{s}} .80,+41^{\circ} 54^{\prime} 10^{\prime}{ }^{\prime} 1(\mathrm{~J} 2000)$ in images taken on 26.48

\footnotetext{
http://www .mpe.mpg.de/ m31novae/opt/m31/index.php
}

Dec. 2008 UT $^{2}$. Liverpool Telescope (LT; Steele et al. 2004) RATCam observations taken on 10.85 Jan. 2009 showed no resolvable objects at the nova position down to a $B$-band limiting magnitude of 19.9 and $B>21.2$ on 13.93 Jan. 2009. The nova was not visible at any other wavebands down to the following limiting magnitudes: 20.1 on $10.85 \mathrm{Jan}$. and 21.2 on $13.93 \mathrm{Jan}$. in $V$-band, 20.8 on 10.84 Jan. and 21.0 on 13.92 Jan. in $r^{\prime}$-band, 19.8 on 10.84 Jan. and 20.9 on 13.92 Jan. in $i^{\prime}$-band.

In 2011 an eruption (M 31N 2011-10e) coincident with the position of M 31N 2008-12a was discovered by Korotkiy and Elenin in data taken on 22.46 Oct. 2011 at an unfiltered magnitude of $18.6 \pm 0.3$, they measured the position as $0^{\mathrm{h}} 45^{\mathrm{m}} 28^{\mathrm{s}} .85$, $+41^{\circ} 54^{\prime} 09^{\prime} .4\left( \pm 00^{\prime} 3\right)$. They reported that no object was visible on 21.35 Oct. to a limiting magnitude of $R>20.0$. Further observations reported the nova to be at unfiltered magnitudes of 18.4 on 22.99 Oct., 19.1 on 23.43 Oct., and >19.7 on 24.47 Oct. Hornoch reported an $R$-band magnitude of $18.18 \pm 0.08$ on 23.12 Oct. $^{3}$. On 26.97 Oct., the nova had a $B$-band magnitude of $20.9 \pm 0.15$ and $V=21.1 \pm 0.16$ (Barsukova et al. 2011).

In 2012, another eruption (M31N 2012-10a) was discovered at $0^{\mathrm{h}} 45^{\mathrm{m}} 28^{\mathrm{s}} .84,+41^{\circ} 54^{\prime} 09^{\prime} .5$ by Nishiyama and Kabashima. They measured the nova to be at an unfiltered magnitude of 18.9 on 18.68 Oct. 2012. The object, which was not visible to a limiting magnitude of 19.8 on 15.52 Oct., appeared to brighten to 18.6 by 19.51 Oct. $^{4}$. The nova was observed at

\footnotetext{
2 http://Www.cbat.eps.harvard.edu/CBAT_M31.html\# $2008-12 \mathrm{a}$

3 http://www.cbat.eps.harvard.edu/unconf/followups/ J00452885+4154094.html

4 http://www. cbat.eps.harvard.edu/unconf/followups/ J00452884+4154095.html
} 


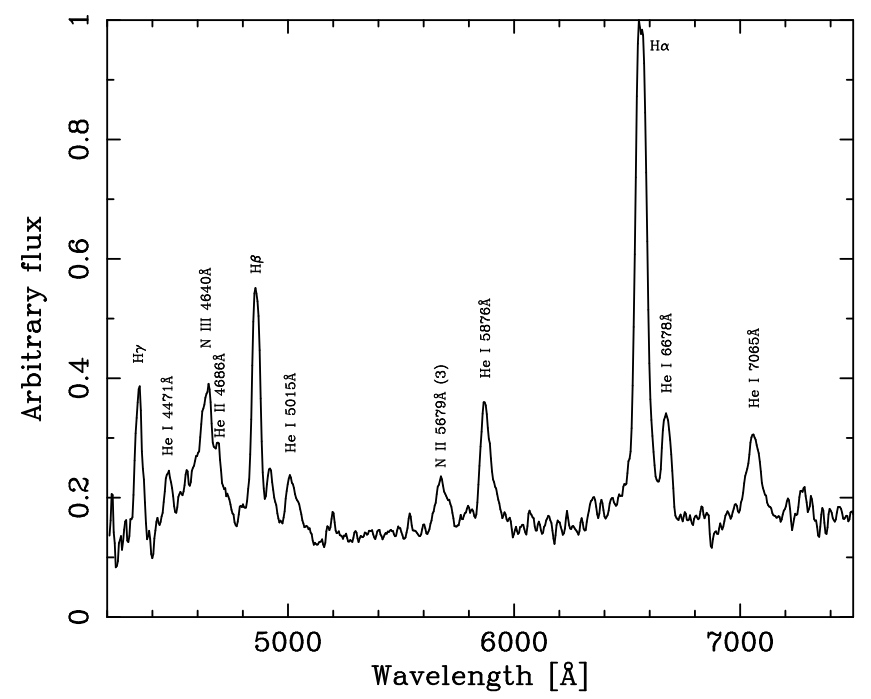

Fig. 1. Hobby-Eberly Telescope LRS spectrum of M31N 2008-12a (truncated at $7500 \AA$ A) taken on 20.34 Oct. 2012 UT, about two days after the 2012 eruption (M 31N 2012-10a).

$R=18.45 \pm 0.04$ on 19.72 Oct. and $i^{\prime}=18.42 \pm 0.06$ on 19.73 Oct. (Shafter et al. 2012). A spectrum of the transient was taken by Shafter et al. (2012, see also Fig. 1) on 20.34 Oct., which was consistent with that of a He/N nova in M 31. The FWHM of the Balmer emission lines indicated an ejecta expansion velocity of $2250 \mathrm{~km} \mathrm{~s}^{-1}$.

\section{Observations of the $\mathbf{2 0 1 3}$ eruption}

The 2013 eruption (M31N 2013-11f) was discovered by the intermediate Palomar transient factor (iPTF) on 27.1 Nov. 2013 UT (ID: PTF09hsd) at $0^{\mathrm{h}} 45^{\mathrm{m}} 28 \mathrm{~s} .89,+41^{\circ} 54^{\prime} 10^{\prime} .2$, subsequently peaking at $R=18.3$ on 28.1 Nov. (Tang et al. 2013). Broadband $B, V$, and $i^{\prime}$ photometry was obtained with the IO:O CCD camera on the LT approximately one and seven days after peak; LT observations are part of a larger programme of photometry and spectroscopy of novae in M31 (see e.g. Shafter et al. 2011). Photometric observations were also obtained using the Danish $1.54 \mathrm{~m}$ telescope at La Silla four and six days after maximum. These data were reduced using standard routines within IRAF (Tody 1993), and calibrated against secondary standards in M 31 (Massey et al. 2006). The photometry is reported in Table 1 and the 2013 lightcurve is presented in Fig. 2.

With limited optical coverage of each eruption, we make the assumption that all eruptions of a RN are essentially the same (Schaefer 2010). We have constructed single $R$ - and $V$-band lightcurves of the eruption using data from the 2008, 2011, 2012, and 2013 eruptions (see Table 1 and Introduction text). These generic lightcurves are represented by the dotted lines in Fig. 2. Based on these generic lightcurves, we estimate that the decline times of this RN are $t_{2}(V) \simeq 4$ days and $t_{2}(R) \simeq 5$ days classifying this RN as very fast. The peak magnitudes observed over these four eruptions are $V=18.4$ and $R=18.18$.

The astrometric position of the 2013 eruption was measured from an LT $i^{\prime}$-band image taken on 28.94 Nov. 2013. An astrometric solution was obtained using 14 stars from the Two Micron All Sky Survey (2MASS) All-Sky Catalogue (Skrutskie et al. 2006) which are coincident with resolved sources in the LT observation. We obtain a position for the 2013 eruption of $\alpha=0^{\mathrm{h}} 45^{\mathrm{m}} 28^{\mathrm{s}} .82 \pm 0.01, \delta=+41^{\circ} 54^{\prime} 10^{\prime}{ }^{\prime} 1 \pm 00^{\prime} .1$ (the astrometric uncertainty is dominated by uncertainties in the plate solution).
Table 1. Observations of the 2013 eruption of M 31N 2008-12a and archival HST observations covering the position of the progenitor.

\begin{tabular}{|c|c|c|}
\hline $\begin{array}{l}\text { JD } \\
2450000+\end{array}$ & $\begin{array}{l}\text { Telescope and } \\
\text { instrument }\end{array}$ & $\begin{array}{l}\text { Photometry } \\
\text { and filter }\end{array}$ \\
\hline 5416.027 & $\mathrm{HST}_{\mathrm{ACS}} / \mathrm{WFC}^{a, b}$ & $F 475 W=24.07 \pm 0.02$ \\
\hline 5415.956 & HST ACS/WFC ${ }^{a, b}$ & $F 814 W=23.90 \pm 0.02$ \\
\hline 5586.715 & HST WFC3/UVIS $^{b}$ & $F 275 W=23.14 \pm 0.06$ \\
\hline 5586.705 & $\mathrm{HST}_{\mathrm{WFC} 3 / \mathrm{UVIS}^{b}}$ & $F 336 W=23.10 \pm 0.03$ \\
\hline 5586.771 & HST WFC3/IR ${ }^{b}$ & $F 110 W>22.05$ \\
\hline 5586.780 & HST WFC $3 / \mathrm{IR}^{b}$ & $F 160 W>21.22$ \\
\hline 5805.013 & HST WFC3/UVIS $^{c}$ & $F 275 W=22.9 \pm 0.1$ \\
\hline 5805.012 & HST WFC3/UVIS ${ }^{c}$ & $F 336 W=22.81 \pm 0.03$ \\
\hline 5805.079 & HST WFC3/IR ${ }^{c}$ & $F 110 W>21.01$ \\
\hline 5805.087 & $\mathrm{HST} \mathrm{WFC} 3 / \mathrm{IR}^{c}$ & $F 160 W>21.18$ \\
\hline 5936.631 & HST ACS/WFC ${ }^{c}$ & $F 475 W=24.49 \pm 0.02$ \\
\hline 5936.538 & HST ACS/WFC ${ }^{c}$ & $F 814 W=24.05 \pm 0.02$ \\
\hline 6625.357 & LT IO:O ${ }^{a}$ & $B=19.51 \pm 0.01$ \\
\hline 6625.425 & LT IO:O ${ }^{a}$ & $B=19.61 \pm 0.01$ \\
\hline 6625.430 & LT IO:O ${ }^{a}$ & $V=19.65 \pm 0.02$ \\
\hline 6625.435 & LT IO:O ${ }^{a}$ & $i^{\prime}=19.29 \pm 0.02$ \\
\hline 6625.519 & Danish 1.54 m DFOSC & $R=19.08 \pm 0.08$ \\
\hline 6625.522 & Danish 1.54 m DFOSC & $V=19.62 \pm 0.09$ \\
\hline 6628.529 & Danish 1.54 m DFOSC & $R=20.0 \pm 0.3$ \\
\hline 6628.533 & Danish 1.54 m DFOSC & $V=20.9 \pm 0.3$ \\
\hline 6628.537 & Danish 1.54 m DFOSC & $I=20.8 \pm 0.3$ \\
\hline 6631.391 & LT IO:O & $B=22.2 \pm 0.3$ \\
\hline 6631.396 & LT IO:O & $V>21.4$ \\
\hline 6636.528 & Danish 1.54 m DFOSC & $R>21.2$ \\
\hline
\end{tabular}

Notes. ${ }^{(a)}$ Williams et al. (2013). ${ }^{(b)}$ Prop. ID: 12056. ${ }^{(c)}$ Prop. ID: 12106.

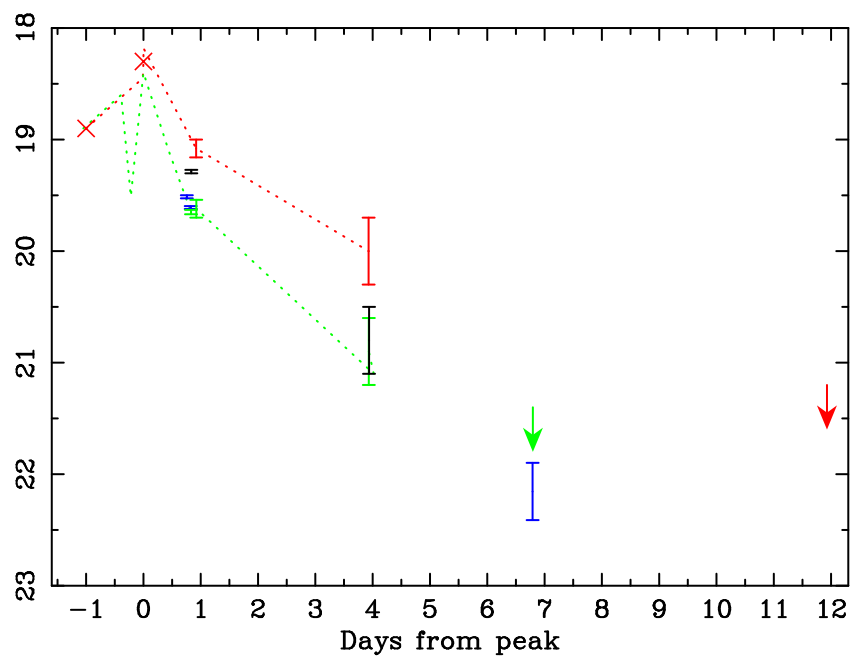

Fig. 2. Optical lightcurve of the 2013 eruption of M31N 2008-12a, data from Tang et al. (2013, $\times$ symbols), LT (3rd and 5th epochs), and Danish $1.54 \mathrm{~m}$ (4th and 6th epochs). Blue data: $B$, green: $V$, red: $R$, and black: $I / i^{\prime}$. Dotted lines indicate a template generic lightcurve based on the $V$ (green) and $R$ (red) observations of the 2008, 2011, 2012, and 2013 eruptions.

A target of opportunity monitoring campaign was also initiated with the Swift satellite (see Henze et al. 2013a,b, and HND2014 for a full discussion).

\section{Progenitor system}

Following the procedure outlined in Bode et al. (2009) and Williams et al. (2014), we undertook a search for any resolved 


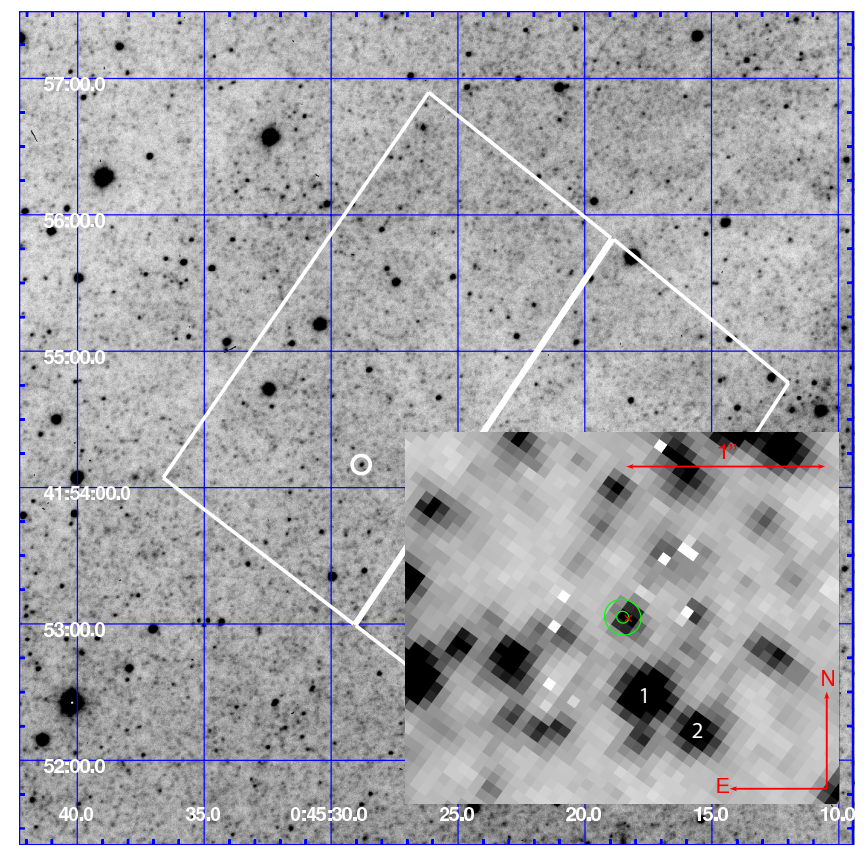

Fig. 3. LT $i^{\prime}$-band image of the 2013 eruption of M31N 2008-12a taken on 28.94 Nov. 2013 UT. The RN position is shown by the white circle; the white boxes indicate the coincident HST ACS/WFC fields. Inset: HST ACS/WFC $F 814 W$ image of the $\sim 2^{\prime \prime} \times 2^{\prime \prime}$ region surrounding M 31N 2008-12a. The inner and outer green ellipses indicate the $1 \sigma$ (31 mas) and $3 \sigma$ radius progenitor search regions, respectively, and the red cross indicates the position of the progenitor candidate. See text for discussion regarding stars 1 and 2.

progenitor system of the 2013 eruption within positionally coincident archival Hubble Space Telescope (HST) data ${ }^{5}$. The position of the 2013 eruption of the RN M 31N 2008-12a was isolated within the archival HST data by calculating a geometric spatial transformation between the LT ( $i^{\prime}$-band; one day post maximum) and HST ACS/WFC F814W data, a method that is independent of the absolute astrometric calibration of the data. This was performed using 16 stars that were visible and unsaturated in both datasets.

There is a resolved object $0.556 \mathrm{ACS} / \mathrm{WFC}$ pixels from the position of the 2013 eruption in the HST ACS/WFC F814W image taken on 7 Aug. 2010 (from proposal ID: 12056; see Fig. 3). This represents a separation of 28 milliarcseconds $(0.9 \sigma)$ from the eruption position. The probability of finding an object at least as close to the eruption position, based on the local resolved stellar density, is only $2.5 \%$. Hence, we can be very confident that the object in the HST data is related to the nova eruption and probably the progenitor/quiescent system.

Hubble Space Telescope archival data were available from two proposals (12056 and 12106); both provided optical F475W and F814W data using ACS/WFC, near-UV F275W and F336W data using WFC3/UVIS, and near-IR F110W and F160W data using WFC3/IR. Photometry of these data was undertaken using DOLPHOT (v2.0; Dolphin 2000, following the standard procedure and parameters given in the manual). The photometry of the candidate progenitor system is reported in Table 1. Whilst the candidate progenitor system was resolved in the optical and NUV HST data, any object at the eruption position would have been severely blended with star 1 (see Fig. 3) in the NIR data. Therefore, we present, as upper limits on the F110W and F160W

\footnotetext{
5 Data taken by the Panchromatic Hubble Andromeda Treasury survey (see e.g. Dalcanton et al. 2012)
}

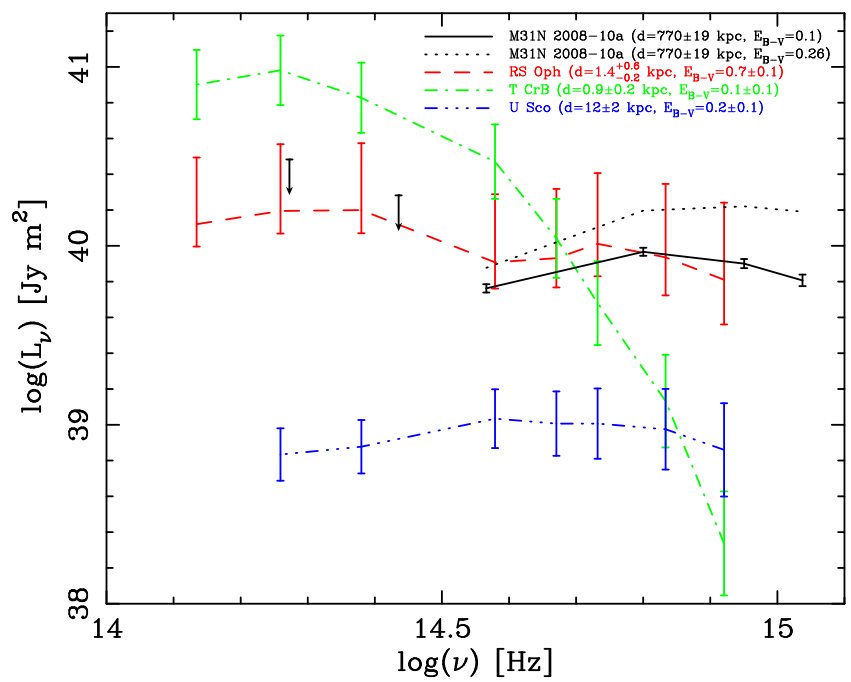

Fig. 4. Distance and extinction-corrected SEDs for the progenitor of M 31N 2008-12a compared to those of the quiescent RNe RS Oph, $\mathrm{T} \mathrm{CrB}$, and U Sco (see Key for line identifications). Units chosen to allow comparison with a similar plot in Schaefer (2010, see their Fig. 71). The black dotted line indicates the maximum effect of any internal M 31 extinction $\left(E_{B-V}^{\text {internal }} \leq 0.16\right)$. For each system, point-to-point uncertainties are small, indicated error bars are dominated by distance and extinction uncertainties.

magnitudes of the candidate progenitor systen, the NIR photometry of star 2. As star 2 is just resolvable from star 1 and is marginally closer to this bright star than the RN, these represent conservative upper limits on the progenitor system brightness.

With multiple waveband observations available a meaningful distance and extinction-corrected spectral energy distribution (SED) can be produced for the quiescent M31N 2008-12a which can be directly compared to that of known Galactic RNe. Figure 4 presents the SED of the quiescent M31N 2008-12a, those of the RG-novae RS Oph and T CrB, and the SG-nova U Sco; we have followed the methodology outlined in Schaefer (2010) to allow direct comparison with their Galactic RN SED (see their Fig. 71). Quiescent photometry for the Galactic RNe is taken from Schaefer (2010, see their Table 30); distances and extinction from Darnley et al. (2012, see their Table 2 and references therein); and optical and NIR absolute calibrations from Bessell (1979) and Campins et al. (1985), respectively. It should be noted that here we use a significantly closer distance to RS Oph, 1. $4_{-0.2}^{+0.6} \mathrm{kpc}$ (Barry et al. 2008; see also Bode 1987). For M31N 2008-12a we assume a distance to M 31 of $770 \pm 19 \mathrm{kpc}$ (Freedman \& Madore 1990), a line-of-sight external (Galactic) reddening of $E_{B-V}=0.1$ (Stark et al. 1992), and additional internal (M 31) reddening of $E_{B-V} \leq 0.16$ (Montalto et al. 2009).

\section{Discussion}

With three eruptions over a two-year period (2011, 2012, and 2013), the RN M $31 N$ 2008-12a is a unique system. Such a short $(\sim 1 \mathrm{yr})$ recurrence time can be expected from a system with a low critical mass for ignition, which requires a highmass WD. Furthermore, to accumulate enough mass for ignition within a short time, a high mass accretion rate is needed. Nova evolution models published by Yaron et al. (2005) indicate that such extremely short recurrence times are possible, but require both a high-mass WD, close to $1.4 M_{\odot}$, and a high mass accretion rate $\left(-8<\log \dot{M} / M_{\odot}<-7\right.$, see their Table 3 and discussion in HND2014). However, the relatively low optical luminosity $\left(V_{\max }=18.4\right)$ and moderate ejecta velocity $\left(2250 \mathrm{~km} \mathrm{~s}^{-1}\right)$ 
are slightly puzzling. The former is discussed in more detail in HND2014 and the derived velocity may be in part due to the inclination of highly shaped ejecta (as can occur in the presence of a massive accretion disk).

Given the eruptions in 2011, 2012, and 2013, each separated by approximately a year, and the eruptions in 2008 and 2009 (Tang et al. 2014) it seems likely that M31N 2008-12a has a recurrence timescale of $\sim 1 \mathrm{yr}$ and that an eruption towards the end of 2010 was missed. The authors HND2014 report on the subsequent X-ray detection following the 2013 eruption, but they also summarise previous X-ray detections at a similar position. Transient X-ray sources were also detected in early 1992, 1993, and late 2001, indicating that this system may have been experiencing yearly eruptions for at least 20 years. The relative faintness of this eruption, its very rapid decline, and its position far out in the disk of M31 may all account for a high number of missed eruptions.

With so many eruptions in such a short time, we must address whether these could be due to spatial coincidence. Following the procedure in Shafter et al. (2011, see their Eq. (6)), the probability of a chance positional coincidence at the location of the nova and within the error circle defined by the reported positions of the 2008, 2011 and 2012 eruptions is just 0.0002 .

The distance and extinction-corrected SED of the quiescent M31N 2008-12a (see Fig. 4) is remarkably similar in the optical to the Galactic RN RS Oph. The SED of RS Oph, with its short ( 20 yr) inter-eruption period, is a combination of the RGB secondary (NIR) and the accretion disk (optical and NUV), unlike that of $\mathrm{T} \mathrm{CrB}$, with a longer recurrence time ( $\sim 80 \mathrm{yr})$, where the SED is dominated by the RGB secondary and there is little sign of a disk. The SEDs of SG-novae, e.g. U Sco, are dominated by the accretion disk with little or no contribution from the less evolved, less luminous, secondary. Given the form and luminosity of the M 31N 2008-12a progenitor SED, it is likely that the progenitor of M31N 2008-12a also contains a significant accretion disk that dominates the NUV and optical flux.

While a RG-nova system similar to RS Oph seems the most likely scenario (based on the SED), a SG-nova system (similar to U Sco) may still be possible. The SED of U Sco is dominated by its accretion disk, but as U Sco is an eclipsing system the disk is observed edge-on, i.e. at its faintest. Given the short recurrence time of M 31N 2008-12a, the observed SED could be due solely to an extremely bright (i.e. very high accretion rate) almost faceon accretion disk. In order to confirm the evolutionary nature of the secondary, stronger limits (or a detection) are needed in the NIR bands, requiring deeper or higher spatial resolution images. Alternatively, the secondary nature could be inferred if the orbital period or inclination of the system can be determined.

The HST archival data is separated into four epochs; the ACS/WFC observations from proposal IDs 12056 and 12106 were taken in Aug. 2010 ( $\sim 14$ mo before the 2011 eruption) and Jan. 2012 ( $\sim 3$ mo after the 2011 eruption and $\sim 9$ mo before the 2012 eruption), respectively. The WFC3 observations were taken in Jan. 2011 and Aug. 2011, 9 and $\sim 2$ mo before the 2011 eruption, respectively. Given the rapid $\left(t_{2}(V) \simeq 4\right.$ days $)$ decline time of the eruption of M31N 2008-12a, all the HST observations are sufficiently distant from any reported eruptions that the system is likely to be near or at quiescence during these observations. That is, we are unlikely to be observing the late decline of any eruption in the HST data. The similarity between the photometry from the two HST datasets implies that even if an eruption in late 2010 has been missed the system was back at quiescence by the end of Jan. 2011.

\section{Conclusions}

The RN M 31N 2008-12a has had five recorded eruptions in the past five years, in 2008, 2009, 2011, 2012, and 2013. Combined data from four of these eruptions indicate a very fast $\mathrm{He} / \mathrm{N}$ nova with a decline time $t_{2}(V) \simeq 4$ days. These observations, coupled with transient X-ray detections in 1992, 1993, and 2001, indicate that this system has a remarkably short $\sim 1$ yr recurrence time. This points to a system containing a very-high-mass WD with a high accretion rate. A search of archival HST data indicates a candidate progenitor system, most likely containing a RGB secondary (RG-nova) and bright accretion disk (e.g. RS Oph), although a SG-nova progenitor (e.g. U Sco) cannot be ruled out.

In addition to this Letter, HND2014 report on the X-ray observations of M31N 2008-12a and a follow-up paper will study the optical and X-ray archives in more detail. The system M31N 2008-12a is unique and we encourage further observations, particularly towards the end of 2014.

Acknowledgements. The LT is operated on the island of La Palma by LJMU in the Spanish Observatorio del Roque de los Muchachos of the Instituto de Astrofisica de Canarias with financial support from STFC. Based (in part) on data collected with the Danish 1.54-m telescope at the ESO La Silla Observatory. S.C.W. acknowledges PhD funding from STFC. M.H. acknowledges support from an ESA fellowship. A.W.S. acknowledges support from NSF grant AST1009566. The work of K.H. and V.V. was supported by project RVO:67985815 and by grant LG12001 of the Ministry of Education of the Czech Republic. Finally, we thank an anonymous referee for the constructive suggestions.

\section{References}

Barry, R. K., Mukai, K., Sokoloski, J. L., et al. 2008, in RS Ophiuchi (2006) and the Recurrent Nova Phenomenon, eds. A. Evans, M. F. Bode, T. J. O'Brien, \& M. J. Darnley (San Francisco, CA: ASP), ASP Conf. Ser., 401, 52

Barsukova, E., Fabrika, S., Hornoch, K., et al. 2011, ATel, 3725

Bessell, M. S. 1979, PASP, 91, 589

Bode, M. F. 1987, RS Ophiuchi (1985) and the Recurrent Nova Phenomenon, ed. M. F. Bode (Utrecht: VNU Science), 241

Bode, M. F., \& Evans, A. 2008, Classical Novae, 2nd edn. eds. M. F. Bode, \& A. Evans (Cambridge University Press), Cambridge Astrophys. Ser., 43

Bode, M. F., Darnley, M. J., Shafter, A. W., et al. 2009, ApJ, 705, 1056

Campins, H., Rieke, G. H., \& Lebofsky, M. J. 1985, AJ, 90, 896

Dalcanton, J. J., Williams, B. F., Lang, D., et al. 2012, ApJS, 200, 18

Darnley, M. J., Bode, M. F., Kerins, E., et al. 2006, MNRAS, 369, 257

Darnley, M. J., Ribeiro, V. A. R. M., Bode, M. F., Hounsell, R. A., \& Williams, R. P. 2012, ApJ, 746, 61

Dolphin, A. E. 2000, PASP, 112, 1383

Freedman, W. L., \& Madore, B. F. 1990, ApJ, 365, 186

Henze, M., Ness, J.-U., Bode, M. F., Darnley, M. J., Williams, S. C. 2013a, ATel, 5627

Henze, M., Ness, J.-U., Bode, M. F., et al. 2013b, ATel, 5633

Henze, M., Ness, J.-U., Darnley, M. J., et al. 2014, A\&A, 563, L8 (HND2014)

Massey, P., Olsen, K. A. G., Hodge, P. W., et al. 2006, AJ, 131, 2478

Montalto, M., Seitz, S., Riffeser, A., et al. 2009, A\&A, 507, 283

Pietsch, W., Haberl, F., Sala, G., et al. 2007, A\&A, 465, 375

Schaefer, B. E. 2010, ApJS, 187, 275

Shafter, A. W., Darnley, M. J., Hornoch, K., et al. 2011, ApJ, 734, 12

Shafter, A. W., Hornoch, K., Ciardullo, J. V. R., Darnley, M. J., \& Bode, M. F 2012, ATel, 4503

Skrutskie, M. F., Cutri, R. M., Stiening, R., et al. 2006, AJ, 131, 1163

Stark, A. A., Gammie, C. F., Wilson, R. W., et al. 1992, ApJS, 79, 77

Steele, I. A., Smith, R. J., Rees, P. C., et al. 2004, Proc. SPIE, 5489, 679

Tang, S., Cao, Y., \& Kasliwal, M. M. 2013, ATel, 5607

Tang, S., Bildsten, L., Wolf, W. M., et al. 2014, ApJ, submitted [arXiv: 1401.2426]

Tody, D. 1993, Astronomical Data Analysis Software and Systems II, ASP Conf. Ser., 52, 173

Williams, S. C., Darnley, M. J., Bode, M. F., \& Shafter, A. W. 2013, ATel, 5611,

Williams, S. C., Darnley, M. J., Bode, M. F., Shafter, A. W., \& Keen, A. 2014 ApJ, submitted

Yaron, O., Prialnik, D., Shara, M. M., \& Kovetz, A. 2005, ApJ, 623, 398 